\title{
Diffusion on the Total Space of a Vector Bundle
}

\author{
Dragoş Hrimiuc
}

\begin{abstract}
In recent years the study of the differential geometry of the total space $E$, of a vector bundle $\pi: R \rightarrow M$, initiated by R.Miron [11], [12] has been developed by many people (see [13] and the references therein). If we take a horizontal complement of the vertical subbundle $V E$, we can express the geometrical objects defined on $E$ in a more simplified form and new geometric objects can be obtained.

Recently P.L.Antonelli and T.Zastawniak in a series of papers [2], [3], [4] extended the Riemannian theory of diffusion processes and stochastic development to the case of Finsler manifolds, the extension being motivated by important problems in Biology [3], [5].

In this paper we extend their formalism to study some geometric problems of the theory of the diffusion process and the stochastic development on $E$, related to these new geometric objects on $E$. We thereby obtain further generalization and geometric meaning for certain results of [2], [3]. But few probabilistic calculations are given here, for they are given in [2], [3], [4]. In a forthcoming publication, as a particular case, the theory of diffusion and stochastic development on Lagrange manifolds will be discussed [9].
\end{abstract}

Mathematics Subject Classification: 60J60, 58G32

Key words: diffusion process, Finsler manifolds, parallel transport

\section{Preliminaries}

Let $\pi: E \rightarrow M$ a smooth vector bundle over $M$. Suppose that $M$ is a real $n$-dimensional differentiable manifold and the dimension of each fibre of $E$ is $m$. Local coordinates on $E$ are $\left(x^{i}, y^{a}\right)$, where $\left(x^{i}\right)$ are local coordinates on the base manifold. Always in this paper, the range for the indices $i, j, k, h, \ldots$ is $\{1,2, \ldots, n\}$, for the indices $a, b, c, d, \ldots$ is $\{1,2, \ldots, m\}$ and the summation convention is used.

A nonlinear connection on $E$ is defined by a distribution $H E$, complementary to the vertical distribution of $T E$, i.e.

$$
T E=H E \oplus V E .
$$

Balkan Journal of Geometry and Its Applications, Vol.1, No.2, 1996, pp. 53-62 (C)Balkan Society of Geometers, Geometry Balkan Press 
A local frame for $T E_{x, y},(x, y) \in E$, adapted to the splitting $(1.1)$ is $\left(\delta_{i}, \delta_{a}\right)$, where

$$
\delta_{i}=\partial_{i}-N_{i}^{a}(x, y) \partial_{a}
$$

is a basis in $H E_{x, y}$ and $\partial_{i}:=\frac{\partial}{\partial x^{i}}, \partial_{a}:=\frac{\partial}{\partial y^{a}}$.

We denote by $\left(d x^{i}, \delta y^{a}\right)$ the dual basis of $\left(\delta_{i}, \partial_{a}\right)$, where

$$
\delta y^{a}=d y^{a}+N_{i}^{a}(x, y) d x^{i} .
$$

Now we can introduce the algebra of $d$-tensor fields on $E$ with respect to the horizontal and vertical distributions. This algebra is locally spanned by $\left(1, \delta_{i}, \partial_{a}\right)$.

A $d$-connection on $E$ is a linear connection on $E$ which preserves by parallelism the horizontal and the vertical distributions (see [13]).

The local coordinate expression for a $d$-connection $\nabla$ on $E$ is given by:

$$
\begin{aligned}
\nabla_{\delta_{i}} \delta_{j}=L_{i j}^{k} \delta_{k}, & \nabla_{\partial_{a}} \delta_{i}=C_{a i}^{j} \delta_{j} \\
\nabla_{\delta_{i}} \partial_{j}=L_{i a}^{b} \partial_{b}, & \nabla_{\partial_{a}} \partial_{b}=C_{a b}^{c} \partial_{c} .
\end{aligned}
$$

A $d$-connection on $E$ determines an $h$ - and $v$-algorithm of covariant derivation. For example if

$$
t=t_{j b}^{i a} \delta_{i} \otimes \delta_{a} \otimes d x^{j} \otimes \delta y^{b}
$$

is a $d$-tensor field on $E$, the horizontal and vertical covariant derivative of $t$ is given by

$$
t_{j b \mid k}^{i a}=\delta_{k} t_{j b}^{i a}+L_{k h}^{i} t_{j b}^{h a}+L_{k c}^{a} t_{j b}^{i c}-L_{k j}^{h} t_{h b}^{i a}-L_{k b}^{c} t_{j c}^{i a}
$$

and

$$
t_{j b \mid c}^{i a}=\partial_{c} t_{j b}^{i a}+C_{c h}^{i} t_{j b}^{h a}+C_{c d}^{a} t_{j b}^{i d}-C_{c j}^{h} t_{h b}^{i a}-C_{c b}^{d} t_{j d}^{i a}
$$

If

$$
G=g_{i j} d x^{i} \otimes d x^{j}+h_{a b} \delta y^{a} \otimes \delta y^{b}
$$

is an $(h, v)$-metric on $E$, these exists $d$-connections compatible with $G$ (see [13], Ch.III).

A systematic presentation of the geometry of $E$ is given in the monograph of Miron and Anastasiei [13].

Throughout this paper we shall use the usual set up for the general theory of stochastic calculus. We follow closely [3], [5]. For an introduction see [6], [7], [10].

Let $(\Omega, \mathcal{F}, P)$ be a probability space endowed with a right continuous filtration $\left(\mathcal{F}_{t}\right)_{t>0}$ such that each $\mathcal{F}_{t}$ contains all negligible events in $\mathcal{F}$. If $f: \Omega \rightarrow R$ is an integrable random variable we denote by $E(f)=\int_{\Omega} f d P$ its expectation and by $E(f / G)$ the conditional expectation of $f$ given $G$. ( $G$ is a sub $\sigma$-field of $\mathcal{F})$.

A stochastic process is a measurable function $x:[0, \infty) \times \Omega \rightarrow R$. One says that a process is continuous if all its sample path, $t \rightarrow x(t, \omega)$, are continuous functions for almost all $\omega \in \Omega$. 
A stochastic process is adapted if for each $t \geq 0$ the random variable $x(t)$ is $\mathcal{F}_{t}$-measurable.

A martingale is an adapted process such that for each $t \geq 0, x(t)$ is integrable and $x(s)=E\left(x(t) / \mathcal{F}_{s}\right)$ for every $t>s \geq 0$.

A continuous local martingale is a continuous adapted process $x$ such that the process

$$
(t, \omega) \rightarrow x\left(\tau_{n} \wedge t, \omega\right) \mathcal{X}_{\left\{0<r_{n}\right\}}(\omega)
$$

is a martingale for every $n$, where $\tau_{n}=\inf \{t \geq 0 ;|x(t)| \geq n\}$ and $\mathcal{X}_{A}$ is the indicator function $A \subset \Omega$.

A continuous $\mathcal{F}_{t}$-adapted process is called a semimartingale if it is written as the sum of a local martingale and a process of bounded variation. For a real semimartingale $x$ and $a$ continuous adapted process $y$ we denote by $y d x:=\int y d x$ the Itô stochastic integral of $y$ with respect to $x, d x d y:=d(x y)-x d y-y d x$ the joint quadratic variation of $x$ and $y$ and $y \circ d x:=\int y_{0} d x$ the Stratonovich stochastic integral, where $y \circ d x=y d x+\frac{1}{2} d x d y$.

If $M$ is a differentiable manifold, a $M$-valued semimartingale is a continuous process $x:[0, \infty) \times \Omega \rightarrow M$ such that $(t, \omega) \rightarrow f(x(t, \omega))$ is a real-valued semimartingale for every smooth function $f: M \rightarrow R$.

If $D$ is an elliptic second-order operator on $M$, an $M$-valued semimartingale $X$ is called a diffusion on $M$ with generator $D$ if the process

$$
(t, \omega) \rightarrow f(x(t, \omega))-f(x,(0, \omega))-\int_{0}^{t} D f(x,(x, \omega)) d s
$$

is a local martingale for every smooth function $f: M \rightarrow R$ with compact support.

\section{Stochastic parallelism on $E$}

We consider an arbitrary smooth curve

$$
c:[0, T] \rightarrow E, \quad c(t)=(x(t), y(t)), \quad t \in[0, T]
$$

locally expressed by the equations:

$$
x^{i}=x^{i}(t), \quad y^{a}=y^{a}(t), \quad t \in[0, T] .
$$

The tangent vector field $\dot{c}$ of $c$ is given by

$$
\dot{c}=\frac{d x^{i}}{d t} \delta_{i}+\frac{\delta y^{a}}{d t} \partial_{a}
$$

If $X=X^{i} \delta_{i}+X^{a} \partial_{a}$ then it is parallel along $c$ if and only if

$$
X_{\mid i}^{j} \frac{d x^{i}}{d t}+\left.X^{j}\right|_{a} \frac{\delta y^{a}}{d t}=0
$$

and 


$$
X_{\mid i}^{b} \frac{d x^{i}}{d t}+\left.X^{b}\right|_{a} \frac{\delta y^{a}}{d t}=0 .
$$

Let $X_{0} \in H_{c(0)} E$ be a horizontal tangent vector at $c(0)$. Since $\nabla$ preserves by parallelism the horizontal distribution we can transport $X_{0}$ by parallelism along $c(t)$ that is, we can find a horizontal vector field $X=X^{i} \delta_{i}$ along $c$, solving of the following system of differential equations:

$$
\frac{d X^{j}}{d t}+L_{k i}^{j} X^{k} \frac{d x^{i}}{d t}+C_{k a}^{j} X^{k} \frac{\delta y^{a}}{d t}=0
$$

with the initial condition

$$
X(0)=X_{0}
$$

The solution of (2.5), (2.6) will be called the $h$-parallel transport of $X_{0}$ along $C$. Analogously, if $\bar{X}_{0}$ is a vertical vector at $c(0)$ the $v$-parallel transport of $\bar{X}_{0}$ along $c$ is defined similarly as the solution of the system of differential equations:

$$
\frac{d \bar{X}^{b}}{d t}+L_{c i}^{b} \bar{X}^{c} \frac{d x^{i}}{d t}+C_{c a}^{b} X^{c} \frac{\delta y^{a}}{d t}=0
$$

with the initial condition:

$$
\bar{X}(0)=X_{0}
$$

From the assumption that the connection is $g$-metrical, if $X(t)$ and $Y(t)$ are two solutions of $(2.5)$ we have

$$
g_{i j}(x(t), y(t)) X^{i}(t) Y^{j}(t)=\text { const. }
$$

Also if $\bar{X}(t), \bar{Y}(t)$ are two solutions of (2.7) we have

$$
h_{a b}(x(t), y(t)) \bar{X}^{a}(t) \bar{Y}^{b}(t)=\text { const. }
$$

In this section we extended the concept of stochastic parallel transport along a trajectory of a diffusion process on $E$. For this, similar to that in [2], [6], [7], we shall approximate the diffusion by piecewise smooth sample path for which the parallel transport is defined by the equations (2.5), (2.6). The theorems below are those of [2], [3], but are here given in a more general geometric setting.

Let $c(t)=(x(t), y(t))$ be a diffusion on $E$ starting from $\left(x_{0}, y_{0}\right)$. If $\pi: 0=$ $t_{0}<t_{1}<\ldots<t_{n}=T$ is a division of the time interval $[0, T]$ we can take a piecewise smooth approximation $c_{\pi}(t)=\left(x_{\pi}(t), y_{\pi}(t)\right)$ on $E$ with bounded first second and third order derivative such that $c_{\pi}\left(t_{\alpha}\right)=c\left(t_{\alpha}\right), \quad \alpha \in\{0,1,2, \ldots, n\}$. If $X_{0} \in H_{\left(x_{0}, y_{0}\right)} E$ the $h$-parallel transport $X_{\pi}(t)$ of $X_{0}$ along $c_{\pi}(t)$ can be defined as a piecewise smooth function, solution of the following system of differential equations:

$$
\frac{d X_{\pi}^{j}}{d t}+L_{k i}^{j}\left(x_{\pi}(t), y_{\pi}(t)\right) X_{\pi}^{k} \frac{d x_{\pi}^{i}}{d t}+C_{k a}^{j}\left(x_{\pi}(t), y_{\pi}(t)\right) X_{\pi}^{k} \frac{\delta y_{\pi}^{a}}{d t}=0
$$




$$
X_{\pi}(0)=X_{0}
$$

We have

Theorem 2.1. The solution of the family of ordinary differential equations (2.11), (2.12) converges in probability as mesh $\pi \rightarrow 0$, to the solution $X(t)$ of the Stratonovich stochastic differential equation

$$
\begin{gathered}
d X^{j}+L_{k i}^{j} X^{k} \circ d x^{i}+C_{k a}^{j} X^{k} \circ \delta y^{a}=0, \\
X(0)=X_{0}
\end{gathered}
$$

where

$$
\delta y^{a}=d y^{a}+N_{i}^{a} \circ d x^{i} .
$$

Definition 2.2. The solution of (2.13), (2.14) is called the stochastic h-parallel transport of $X_{0}$ along the diffusion $c$.

Similarly we shall be able to define the $v$-stochastic parallel transport of $\bar{X}_{0} \in V E_{c(0)}$ along a diffusion $c$ as the solution of the Stratonovich stochastic differential equation

$$
\begin{gathered}
d \bar{X}^{b}+L_{c i}^{b} \bar{X}^{c} \circ d x^{i}+C_{c a}^{b} \bar{X}^{c} \circ \delta y^{a}=0, \\
\bar{X}(0)=\bar{X}_{0} .
\end{gathered}
$$

Remark 2.3. Let $c(t)=(x(t), y(t))$ be a diffusion on $E$ starting at $\left(x_{0}, y_{0}\right)$. If $X_{0}=X^{i} \frac{\partial}{\partial x^{i}} \in T M_{x_{0}}$ and $A_{0}=A^{a} s_{a} \in E_{x_{0}}$ we can take the horizontal and vertical lift of $X_{a}$ and $A_{0}$

$$
X_{0}^{v}=X^{i} \frac{\delta}{\delta x^{i}} \quad \text { and } \quad A_{0}^{v}=A^{a} \frac{\partial}{\partial y^{a}} .
$$

Now, we can define the stochastic $h$ - and $v$-parallel transport for $X_{0}$ and $A_{0}$ along the diffusion $c$ on $E$ as the stochastic $h$ - and $v$-parallel transport for $X_{0}^{v}$ and $A_{0}^{v}$.

Theorem 2.4. (i) If $X(t)$ and $Y(t)$ are any two solutions of (2.13) then

$$
g_{i j}(x(t), y(t)) X^{i}(t) X^{j}(t)=\text { const.a.s. }
$$

(ii) If $\bar{X}(t)$ and $\bar{Y}(t)$ are any two solutions of (2.16) then

$$
h_{a b}(x(t), y(t)) \bar{X}^{a}(t) \bar{Y}^{b}(t)=\text { const.a.s. }
$$

Let $c:[0, T] \rightarrow E$ be a diffusion on $E, c(t)=(x(t), y(t))$. We say that $c$ is a horizontal diffusion if $y(t)$ is a solution of the following stochastic differential equation

$$
d y^{a}+N_{i}^{a}(x, y) \circ d x^{i}=0, \quad y(0)=y_{0}
$$


Also we say that $c$ is a vertical diffusion if $x(t)=x_{0}$ a.s. The system (2.21) has, generally a local solution. We cannot, in general, extend the solution for almost all $t \in[0, T]$ but there are some important situations in which we can do it [2], [3], [9]. We can give a geometric description of the solution of (2.21).

Let $x:[0, T] \rightarrow M$ be a diffusion on $M$ starting from $x_{0}$ and $x_{\pi}$ a piecewise smooth approximation with bounded first, second and third order derivative, associated to the division $\pi: 0=t_{0}<t_{1}<\ldots<t_{n}=T$.

We define the nonlinear transport by parallelism of $y_{\pi}$ along $x_{\pi}$ as the solution of the following (nonlinear) system of ordinary differential equations

$$
\frac{d y_{\pi}^{a}}{d t}+N_{i}^{a}\left(x_{\pi}, y_{\pi}\right) \frac{d x_{\pi}^{i}}{d t}=0, \quad y_{\pi}(0)=y_{0}
$$

Theorem 2.5. The solution of (2.22) converges in probability as mesh $\pi \rightarrow 0$ to the solution of $y(t)$ of the Stratonovich stochastic equation (2.21).

Remark 2.6. If the solution of system (2.22) is defined on $[0, T]$, the same is valid for the solution of the stochastic differential system (2.21).

Remark 2.7. If $c:[0, T] \rightarrow E, \quad c(t)=(x(t), y(t))$ is a horizontal diffusion then the equations $(2.13),(2.14)$ and $(2.16),(2.17)$ are written:

$$
d X^{j}+L_{k i}^{j} X^{k} \circ d x^{i}=0, \quad X(0)=X_{0}
$$

and

$$
d \bar{X}^{b}+L_{c i}^{b} \bar{X}^{c} \circ d x^{i}=0, \quad \bar{X}(0)=\bar{X}_{0} .
$$

If $c(t)=(x(t), y(t))$ is a vertical diffusion the equations (2.13), (2.14) and (2.16), (2.17) become

$$
d X^{j}+C_{k a}^{j} X^{k} \circ d y^{a}=0, \quad X(0)=X_{0}
$$

and

$$
d \bar{X}^{b}+C_{c a}^{b} \bar{X}^{c} \circ d y^{a}=0, \quad \bar{X}(0)=\bar{X}_{0} .
$$

\section{Stochastic development on $\mathrm{E}$}

Let $O^{\prime}(E)$ be the principal bundle of frames on $E$ defined as follows. The total space of $O^{\prime}(E)$ consists of elements $(x, y, z)$, where $x \in M, \quad y \in E_{x}$ and $z=\left(e_{1}, \ldots, e_{n}, \bar{e}_{1}, \ldots, \bar{e}_{m}\right)$ is frame of $T E_{(x, y)}$ such that $\left(e_{1}, \ldots, e_{n}\right)$ is an orthogonal frame of $H E_{(x, y)}$ relative to the metric structure $g$ and $\left(\bar{e}_{1}, \ldots, \bar{e}_{m}\right)$ is an orthogonal frame in $V E_{(x, y)}$ with respect to the metric structure $h$.

The differential structure of $O^{\prime}(E)$ can be obtained from that of $E$ as follows.

Let us consider $\left(U_{\alpha}, \Phi_{\alpha}\right)$ be a coordinate system of $E$ and $\tilde{U}_{\alpha}=\{(x, y, z) \in$ $O^{\prime}(E) ; \quad(x, y) \in U_{\alpha}$ and $z$ is a frame as above $\}$.

We define the mapping:

$$
\tilde{\Phi}_{\alpha}: \tilde{U}_{\alpha} \rightarrow \Phi_{\alpha}\left(\tilde{U}_{\alpha}\right) \times O(n) \times O(m) \subset R^{n+m} \times R^{n^{2}+m^{2}}
$$

by 


$$
\tilde{\Phi}(x, y, z)=\left(\Phi_{\alpha}(x, y), e_{j}^{i}, \bar{e}_{b}^{a}\right)
$$

where

$$
e_{j}=e_{j}^{i}\left(\frac{\delta}{\delta x^{i}}\right)_{(x, y)}, \quad \bar{e}_{b}=\bar{e}_{b}^{a}\left(\frac{\partial}{\partial y^{a}}\right)_{(x, y)}
$$

and $O(n)$ is the group of orthogonal transformations in $R^{n}$. The projection $\pi: O^{\prime}(E) \rightarrow E$ is defined as usual by $\pi(x, y, z)=(x, y)$ and the right action by $R_{v}(x, y, z)=(x, y, z \cdot v)$, where

$$
z \cdot v=\left(e_{i} u, \bar{e}_{a} \bar{u}\right), \quad e_{i} u=u_{i}^{j} e_{j}, \quad \bar{e}_{a} \bar{u}=\bar{u}_{a}^{b} \bar{e}_{b}
$$

for any $v=(u, \bar{u}) \subset O(n) \times O(m)$ and $(x, y, z) \subset O^{\prime}(E)$.

If $\alpha(t)=\left(\gamma^{i}(t), \bar{\gamma}^{a}(t)\right), \quad t \in[0, T]$ is a smooth curve in $R^{n+m}$ using the $d$-connection $\nabla$ we cal roll $E$ along $\alpha(t)$ to obtain a curve $c(t)$ on $E$ as a trace of $\alpha(t)$. In fact, if $\left(x_{0}, y_{0}, z_{0}\right) \in O^{\prime}(E)$ we must find a smooth curve $\tilde{c}(t)=$ $(x(t), y(t), z(t))$ on $O^{\prime}(E)$ such that

$$
\begin{gathered}
\frac{d x^{i}}{d t}=\frac{d \gamma^{j}}{d t}(t) e_{j}^{i}(t), \quad \frac{\delta y^{a}}{d t}=\frac{d \bar{\gamma}^{b}}{d t} \bar{e}_{b}^{a}(t), \\
\frac{d e_{h}^{j}}{d t}+L_{k i}^{j} e_{h}^{k} \frac{d x^{i}}{d t}+C_{k a}^{j} e_{h}^{k} \frac{\delta y^{a}}{d t}=0, \\
\frac{d \bar{e}_{d}^{b}}{d t}+L_{c i}^{b} \bar{e}_{d}^{c} \frac{d x^{i}}{d t}+C_{c a}^{b} \bar{e}_{d}^{c} \frac{\delta y^{a}}{d t}=0, \\
x^{i}(0)=x_{0}^{i}, \quad y^{a}(0)=y_{0}^{a}, \quad e_{i}(0)=e_{0 i}, \quad \bar{e}_{a}(0)=\bar{e}_{0, a} .
\end{gathered}
$$

For the curve $c(t)=c\left(t, x_{0}, y_{0}, z_{0}, \alpha\right)=\pi(\tilde{c}(t))$ it follows:

$$
c\left(t, x_{0}, y_{0}, z_{0} \cdot v, \alpha\right)=c\left(t, x_{0}, y_{0}, z_{0}, v \cdot \alpha\right), \quad t \in[0, T],
$$

where $v=(u, \bar{u}) \in O(n) \times O(m)$ and $v \cdot \alpha$ is the curve in $R^{n+m}$ defined by

$$
(v \cdot \alpha)(t)=\left(u_{j}^{i} \gamma^{j}(t), \bar{u}_{b}^{a} \bar{\gamma}^{b}(t)\right)
$$

Let $c(t)=(x(t), y(t)), \quad t \in[0, T]$ be a diffusion on $E$ starting at $\left(x_{0}, y_{0}\right)$ and $z_{0}$ an orthogonal frame in $T E_{\left(x_{0}, y_{0}\right)}$ as above.

We use the stochastic parallel transport to move this orthogonal frame along $c(t)$ and we shall obtain the moving frame $z(t)=\left(e_{1}(t), \ldots, e_{n}(t), \bar{e}_{1}(t), \ldots, \bar{e}_{m}(t)\right)$ such that the following stochastic differential equations are satisfied:

$$
\begin{array}{r}
d e_{h}^{j}+L_{k i}^{j} e_{h}^{k} \circ d x^{i}+C_{k a}^{j} e_{h}^{k} \circ \delta y^{a}=0, \\
d \bar{e}_{d}^{b}+L_{c i}^{b} \bar{e}_{d}^{c} \circ d x^{i}+C_{c a}^{b} \bar{e}_{d}^{c} \circ \delta y^{a}=0
\end{array}
$$

and almost surely on $[0, T]$ we have:

$$
\begin{aligned}
& g_{i j}(x(t), y(t)) e_{h}^{i}(t) e_{k}^{j}(t)=\delta_{h k}, \\
& h_{a b}(x(t), y(t)) e_{c}^{a}(t) e_{d}^{b}(t)=\delta_{c d} .
\end{aligned}
$$
$O^{\prime}(E)$.

We regard $x(t), y(t), z(t))$ as a stochastic process on the orthogonal bundle

Now, we can study the concept of stochastic development on $E$ or rolling the total space $E$ along a standard Brownian motion in $R^{n+m}$, extending the Riemannian and Finslerian stochastic development of [2], [3]. 
Let $w(t), v(t)$ be two independent standard Brownian motion in $R^{n}$ and $R^{m}$, thus $(w(t), v(t))$ is a standard Brownian motion in $R^{n+m}$.

The system of stochastic differential equations for the stochastic development on $E$ can be written as:

$$
\begin{gathered}
d x^{i}=e_{j}^{i} \circ d w^{j} ; \quad \delta y^{a}=\bar{e}_{b}^{a} \circ d v^{b}, \\
d e_{h}^{j}+L_{k i}^{i} e_{h}^{k} \circ d x^{i}+C_{k a}^{j} e_{h}^{k} \circ \delta y^{a}=0, \\
d \bar{e}_{d}^{b}+L_{c i}^{b} \bar{e}_{d}^{c} \circ d x^{i}+C_{c a}^{b} \bar{e}_{d}^{c} \circ \delta y^{a}=0, \\
x^{i}(0)=x_{0}^{i}, \quad y^{a}(0)=y_{0}^{a}, \quad e_{i}(0)=e_{0 i}, \quad \bar{e}_{a}(0)=\bar{e}_{0 a},
\end{gathered}
$$

with $\left(x_{0}, y_{0}, z_{0}\right) \in O^{\prime}(E)$.

From (3.5) it follows that the solution of (3.6) is a process on $O^{\prime}(E)$.

Definition 3.1. The solution $(x(t), y(t), z(t))$ of $(3.6)$ is called it the stochastic development on $E$.

Theorem 3.2. The solution of stochastic differential equation (3.6) defines a flow diffeomorphisms on $O^{\prime}(E)(x(t), y(t), z(t))$, whose projection $(x(t), y(t))$, from $O^{\prime}(E)$ to $E$ is a diffusion on $E$ starting at $\left(x_{0}, y_{0}\right)$ which have the probability law independent of the choice of the initial orthonormal frame $z_{0}$ in $E_{\left(x_{0}, y_{0}\right)}$ and whose generator is

$$
D=\frac{1}{2} g^{i j}\left(\delta_{i} \delta_{j}-L_{i j}^{k} \delta_{k}\right)+\frac{1}{2} h^{a b}\left(\partial_{a} \partial_{b}-C_{a b}^{c} \partial_{c}\right) .
$$

This result is that of [2], [3] but is here given a more general geometric setup. The reader should consult these papers for proof of (3.7).

\section{Examples}

We consider the tangent bundle $\pi: T M \rightarrow M$ with the $(h, v)$-metric

$$
G=g_{i j} d x^{i} \otimes d x^{j}+h_{a b} \delta y^{a} \otimes \delta y^{b}
$$

$G$ is called $h$-Riemannian ( $v$-Riemannian) if its horizontal (vertical) part $g_{i j}(x, y) \quad\left(h_{a b}(x, y)\right)$ are functions of position only, i.e. depend on $x$ alone. If $G$ is $h$ - and $v$-Riemannian we shall say that $G$ is $(h, v)$-Riemannian. Also, we say that $G$ is locally-Minkowski if locally $h_{a b}(x, y)=h_{a b}(y)$. The $v$-metric $h_{a b}$ is called weakly regular if the $d$-tensor field $\tilde{h}_{a b}=\frac{1}{2} \partial_{a} \partial_{b} \epsilon$ is nondegenerate, where $\epsilon=h_{a b}(x, y) y^{a} y^{b}$ (see [13]).

1. We suppose $G$ is Riemannian-locally Minkowski metric on $T M$ and $h_{a b}(y)$ is weakly regular. Then we can take $N_{i}^{a}=0$ (sse [13], page 126) and the coefficients of the canonical $d$-connection compatible with $G$ are given by

$$
\begin{gathered}
L_{j k}^{i}=\gamma_{j k}^{i}(x)=\frac{1}{2} g^{i h}\left(\delta_{k} g_{h j}+\delta_{j} g_{h k}-\delta_{h} g_{j k}\right), \quad L_{i b}^{a}=0 \\
C_{c j}^{i}=0 ; \quad C_{b c}^{a}=\frac{1}{2} h^{a d}\left(\partial_{b} h_{d c}+\partial_{c} h_{d b}-\partial_{d} h_{b c}\right) .
\end{gathered}
$$

The system (3.6) for the stochastic development takes the form 


$$
\begin{array}{ll}
d x^{i}=e_{j}^{i} \circ d w^{j} ; & d e_{h}^{i}+\gamma_{k i}^{j} e_{h}^{k} \circ d x^{i}=0 \\
d y^{a}=\bar{e}_{b}^{a} \circ d v^{b} ; & d \bar{e}_{d}^{b}+C_{c a}^{b} \bar{e}_{d}^{c} \circ d y^{a}=0 .
\end{array}
$$

2. Let $G$ be a $(h, v)$-Riemannian metric. Then we can take $N_{i}^{a}=\gamma_{i b}^{a} y^{b}$ as a nonlinear connection on $T M\left(\gamma_{j k}^{i}\right.$ are the Christoffel symbols formed with $\left.g_{i j}\right)$. The coefficients of the canonical $d$-connection, compatible with $G$ are given by

$$
L_{j k}^{i}=\gamma_{j k}^{i} ; \quad L_{b k}^{a}=\gamma_{b k}^{a}+\frac{1}{2} h^{a c} h_{b c \| k} ; \quad C_{j k}^{i}=0, \quad C_{b c}^{a}=0
$$

where $h_{a b \| k}$ denote the covariant derivative of $h_{a b}$ with respect to $\gamma_{j k}^{i}$. The system (3.6) for the stochastic development reads:

$$
\begin{gathered}
d x^{i}=e_{j}^{i} \circ d w^{j} \\
d e_{h}^{j}=\gamma_{k i}^{i} e_{h}^{k} \circ d x^{i}=0 \\
\delta y^{a}=\bar{e}_{b}^{a} \circ d v^{b} \\
d \bar{e}_{d}^{b}+\left(\gamma_{a i}^{b}+\frac{1}{2} h^{b c} h_{a c \| i}\right) \bar{e}_{d}^{a} \circ d x^{i}=0 .
\end{gathered}
$$

Acknowledgements. The author would like to thank the Department of Mathematical Sciences at the University of Alberta for their assistance during the preparation of this paper. Also, thanks are due for P.Antonelli's Grant NSERCA 7667 which helped defer the author's expenses during his 1994-95 stay in Edmonton. Finally, the author would like to thank Vivian Spak for her excellent typesetting.

\section{References}

[1] M.Anastasiei, Vector bundle. Einstein equations, An. Şt. Univ. Iaşi, s I a Mat, 1986, 17-24.

[2] P.L.Antonelli and T.J.Zastawniak, Diffusions on Finsler manifolds, Proc. of the XXV-th Symposium on Mathematical Physics, Torun, Poland, 1992.

[3] P.L.Antonelli and T.J.Zastawniak, Stochastic calculus on Finsler manifolds and an application in biology, to appear in Nonlinear World, 1993.

[4] P.L.Antonelli and T.J.Zastawniak, Diffusion on the tangent and indicatrix bundle on a Finsler manifold, Tensor, N.S., 56(1995), 233-247.

[5] P.L.Antonelli and T.J.Zastawniak (editors), Lagrange Differential Geometry, Finsler Spaces and Diffusions Applied in Biology and Physics, Pergamon Press, 1994, vol.20, Math. and Compt. Mod.

[6] K.D.Elworthy, Stochastic Differential Equations on Manifolds, Cambridge University Press, Cambridge, 1982. 
[7] M.Emery, Stochastic Calculus in Manifolds, Springer-Verlag, Berlin, Heildelberg, 1989.

[8] D.Hrimiuc, On the geometry of the total space of an infinite dimensional vector bundle, An. Şt. Univ. "Al.I.Cuza", Iaşi 35, 1989, 77-78.

[9] D.Hrimiuc, Diffusion on Lagrange manifolds, (to appear).

[10] H.Ikeda and S.Watanabe, Stochastic Differential Equations and Diffusion Processes, North Holland, Amsterdam, Kodansha, Tokyo, 1989.

[11] R.Miron, Introduction to the theory of Finsler spaces, Proc. of the Nat. Seminar on Finsler spaces, Braşov, 1980, 131-183.

[12] R.Miron, Vector bundles Finsler geometry, Proc. of the Nat. Seminar on Finsler spaces, Braşov, 1982, 147-188.

[13] R.Miron and M.Anastasiei, The Geometry of Lagrange Spaces, Theory and Applications, Kluwer Academic Press, FTPH, No.59, 1994.

University Al.I. CUZA,

Department of Mathematics, Iaşi, 6600, Romania 